\title{
ESTRUCTURA DE LA SIGNIFICACIÓN EN LOS EMPEÑOS DE UNA CASA, DE SOR JUANA INÉS DE LA CRUZ
}

\author{
Jorge Urrutia \\ Universidad de Sevilla
}

Marcelino Menéndez Pelayo, en su Historia de la poesía Hispanoamericana, tildaba de «linda» la comedia de Sor Juana Inés de la Cruz Los empeños de una casa. La definía también como «interesante y gallarda imitación (...) de las comedias de capa y espada de Calderón». Es sabido que Sor Juana tuvo, efectivamente, influencia del principal dramaturgo barroco español. Escrita su comedia en 1683, dos años después de morir éste, en varias ocasiones demuestra su fervor por él. Así, en la Jornada tercera, el criado Castaño, inmerso en una dificultad, busca:

... alguna traza

que de Calderón parezca

con que salir de este empeño (v. 2405-2407) ${ }^{1}$.

Dicha traza va a consistir en disfrazarse con unos vestidos de mujer, lo que le acarrea nuevos problemas, del mismo modo en que al criado de una comedia de Calderón le dificulta la vida cierto traje, haciéndole exclamar:

...j Ay vestido

en qué confusión me has puesto! (Ja I)

La cita corresponde a una comedia, probablemente de 1639 , que suele atribuirse a Calderón y se titula Los empeños de un acaso. Sor Juana parodia el título de Calderón a través de la similitud fonética: Los empeños de un acaso / Los

1 Cito por la edición de Carlos Ripoll y Andrés Valdespino: Teatro hispanoamericano. Antología crítica; tomo I; «época colonial»; New York; Anaya Book Co. Onc., 1972, que numera los versos. La edición completa del texto (incluyendo loa, sainetes y sarao) la sigo en Obras selectas de Sor Juana de la Cruz (prólogo, selección y notas de Georgina Sabat de Rivers y Elías Rivers); Barcelona; Noguer, 1976. 
empeños de una casa. No es ésa la única parodia que la monjita mejicana hace del sacerdote español.

Una comedia del Siglo de Oro no se representaba aislada, sino que, como es sabido, iba precedida de una loa, intercalaba entre dos jornadas un entremés, sainete o paso y concluía con un sarao. No siempre todas y cada una de tales piezas pertenecían al mismo autor ni las conocemos. En el caso de Los empeños de una casa, Sor Juana las escribió todas y nos han sido conservadas.

En el segundo de los entremeses intercalados, un autor de comedias denominado Acevedo, entre los silbidos que los espectadores parecen dedicar a su representación, dice:

¡Ay silbado de mí! ¡Ay desdichado! ¿Que la comedia que hice me han silbado! ¿Al primer tapón silbos? Muerto quedo.

Parodia clara del primer verso del famoso monólogo de Segismundo en La vida es sueño:

¿Ay mísero de mí! ¡Ay infelice!

Pero la obra de Sor Juana Inés de la Cruz no puede verse tan sólo desde el prisma calderoniano. Tiene importancia por sí misma y es exponente de unos planteamientos estéticos y sociales que, si no renuevan el género dramático bajo cuyo código se expresan, sí ofrecen objeto serio para la reflexión.

Enrique Anderson Imbert considera la obra divertidísima y afirma que «se adivina la sonrisa burlona de Sor Juana ante la necedad de hombres y mujeres que creerr que es posible forzar el amor. Don Pedro ama a Doña Leonor, pero Doña Leonor ama a Don Carlos. Don Juan ama a Doña Ana, pero Doña Ana ama a Don Carlos. Al final sólo es feliz la pareja de amantes sinceros: el buen Don Carlos y la discreta y hermosa Doña Leonor». Recordemos, pues, el argumento.

DON PEDRO está enamorado de DOÑA LEONOR sin que ella lo sepa. DO$\tilde{N} A$ ANA está enamorada de DON CARLOS, ajeno totalmente a ello, y pretende interrumpir las relaciones con DON JUAN.

DOÑA LEONOR va a fugarse con DON CARLOS. Enterado DON PEDRO, hace que unos hombres de armas persigan al caballero y depositen a la dama junto a DOÑA ANA; piensa así poder cortejarla con facilidad. En la casa coinciden sin saberlo: DON PEDRO, DOÑA ANA y su criada -CELIA-, DOÑA LEONOR, DON CARLOS - que se ha refugiado allí huyendo de los perseguidores con su criado CASTAÑO y ha sido admitido por DOÑ $A$ ANA - y DON JUANque ha convencido a la criada para que le deje hablar con DOÑA ANA.

El PADRE de Doña Leonor, enterado de dónde está su hija, cree que DON PEDRO es el raptor. Le pide que se case con ella, a lo que él accede. DON CARLOS asiste escondido a la conversación pero no puede descubrirse por no comprometer a DOÑA ANA; decide enviar a su criado a explicarle todo al PADRE de Doña Leonor. CASTAÑ̃ se viste, para no ser descubierto, con unas ropas de doña Leonor que guardaba desde la frustada huída y es confundido con ella por DON PEDRO. Éste se declara y le hace prometer que se casará con él. 
Prosiguen los equívocos y las escenas en tinieblas o a media luz. Tras una de éstas, DOÑA ANA declara querer casarse con el hombre que trae de la mano y que cree DON CARLOS, pero es DON JUAN. DON PEDRO, por su parte insiste en casarse con la mujer que escondió en un cuarto y resulta ser CASTAÑO disfrazado y no DOÑA LEONOR. Termina la comedia con las bodas de DON CARLOS y DOÑA LEONOR y de DON JUAN y DOÑA ANA, así como con la de CASTAÑO Y CELIA.

Para llegar, si es posible, a un mejor entendimiento de la obra intentará aplicar el método de análisis que propone Algirdas Julien Greimas, ya en su Semántica estructural aunque tiene algunas ampliaciones posteriores ${ }^{2}$, para diseñar la estructura elemental de la significación.

Según Greimas, la significación puede considerarse primeramente como un eje limitado por dos términos contrarios:

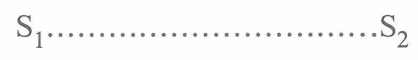

Por ejemplo:

$$
\text { bueno....................malo }
$$

Cada uno de estos contrarios tiene su correspondiente término contradictorio, pudiendo ambos constituir otro eje:

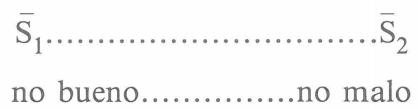

Si buscamos expresar también las relaciones de implicación que se establecen, es posible trazar el siguiente modelo del sistema elemental de la significación:

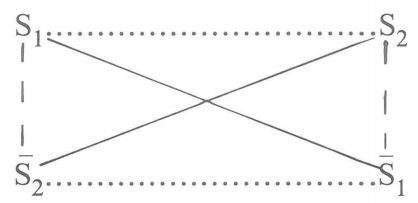

Donde la línea de puntos [.....] expresa la contrariedad, la línea continua [__ expresa la contradicción y la línea discontinua [-------] expresa la implicación.

8 A. J. Greimas; Semántique structurale: París; Larousse, 1966 (hay traducción española en Madrid: Gredos). A. J. Greimas: Du Sens; París: Seuil, 1970 (hay traducción española, Sobre el sentido, con interesantes modificaciones en Madrid: Fragua, 1973). Se amplía a través de la teoría de las modalidades en Du sens II; París: Seuil, 1983 (hay traducción italiana con un prólogo muy útil en Milano: Bompiani, 1985). Véanse también, entre otros libros; J. Courtés: Introduction à la sémiotique narrative et discursive (París: Hachette, 1976); David Maldavsky: Teoría literaria general (Buenos Aires: Paidós, 1974); Grupo de Entrevernes: Análisis semiótico de los textos (Madrid: Cristiandad, 1982). 
Con el ejemplo ya usado:

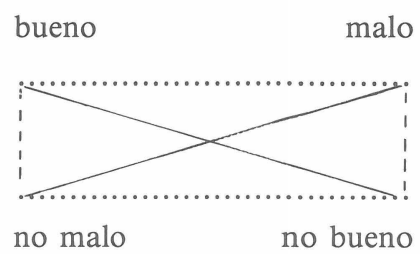

Greimas, como ejemplo, propone el sistema de las relaciones sexuales en la sociedad francesa. Vamos a tomarlo nosotros y a aplicarlo a Los empeños de una casa.

Dice Greimas que las relaciones sexuales obedecen a un código: la sociedad las regula mediante la prescipción de las relaciones matrimoniales y la prohibición de las relaciones anormales. Hay, pues, dos ejes de contrarios:

$$
\begin{gathered}
\text { relaciones matrimoniales / relaciones no matrimoniales } \\
\text { relaciones normales / relaciones anormales }
\end{gathered}
$$

Tracemos el esquema:

\section{RELACIONES PERMITIDAS}

prescritas: matrimoniales

$\mathrm{M}_{1}$

\section{RELACIONES EXCLUIDAS}

prohibidas: anormales

(ej: incesto, homosexualidad)

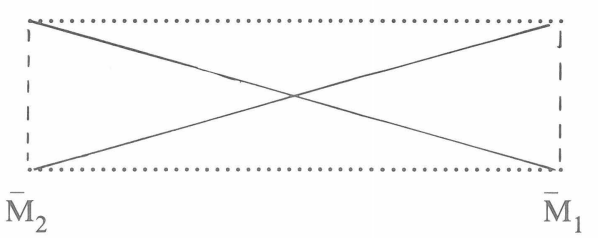

no prohibidas: normales

no prescritas:

no matrimoniales

(ej: adulterio masculino)

(ej: adulterio femenino)

¿Cuáles son las relaciones que, en Los empeños de una casa, corresponden a $\mathrm{M}_{1}$ ? Evidentemente los diversos matrimonios que se dan en la obra: Don Carlos / Doña Leonor, Don Juan / Doña Ana y Castaño / Celia.

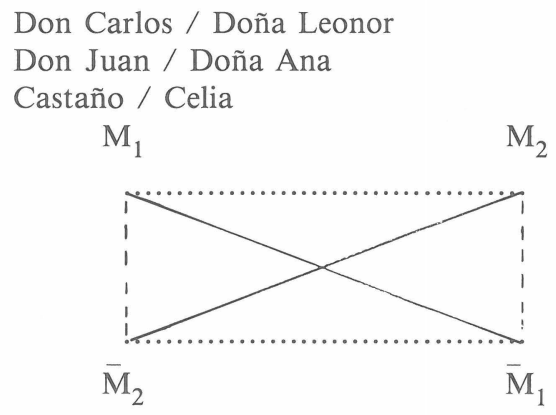


Pero a esa situación se llega al cabo de la tercera jornada. Podremos matizar más.

En $\mathrm{M}_{2}$ podemos situar aquellas relaciones que no sólo están excluidas socialmente, sino que están expresamente prohibidas, como serían las incestuosas, las homosexuales o las que atacan a la jerarquía social (las amorosas que se dieran entre criados y señores, por ejemplo). Ninguna de estas tres posibilidades se dan plenamente en la obra.

Como es habitual en el teatro del siglo de Oro, deja la autora muy clara la calidad social de los personajes y ello tiene importancia primordial. Un personaje de El mercader amante, comedia de Gaspar de Aguilar, explica que le es muy difícil decidir entre dos mujeres:

$$
\begin{aligned}
& \text { siendo como son, las dos, } \\
& \text { tan iguales en estado, } \\
& \text { en linaje y discreción } \\
& \text { en riqueza y en bondad }
\end{aligned}
$$

En Los empeños de una casa se destaca que los personajes principales son todos ellos nobles. Los matrimonios no van, en ningún caso, a atentar contra la ordenación social. Así, Don Juan advierte a Don Pedro:
Don Pedro, vos no ignoráis
como tan gran caballero,
las muchas obligaciones
que tenéis que parecerlo. (v. 1861-4)

Doña Ana, como hermana de Don Pedro, tiene igual calidad social. Y a Don Pedro no le parece poca la alcurnia de Don Juan, puesto que dice:

$$
\begin{aligned}
& \text {... Yo doy por bien } \\
& \text { la burla que se me ha hecho, } \\
& \text { porque se case mi hermana } \\
& \text { con Don Juan. (v. 3361-4) }
\end{aligned}
$$

Leonor cuenta de sí misma:

Yo nací noble; éste fue de mi mal el primer paso, que no es pequeña desdicha nacer noble un desdichado. (v. 271-4)

Y de Don Carlos:

...llegó acaso a verme...

Don Carlos de Olmedo, un joven

forastero, mas tan claro por su origen, que en cualquiera lugar que llegue a hospedarlo, podrá no ser conocido pero no ser ignorado. (v. 389-393/8)

3 Citado por José Antonio Maravall: «El principio del honor en la sociedad tradicional»; en Ideologies and Literature, vol. II, n. 7, May-June, 1978, pág. 12. 
Hay una relación homosexual que no llega a cumplirse, pero que permite al quedar flotante en una confusión- mantener un cierto equívoco. Me refiero a la relación que se establece entre Don Pedro y el criado Castaño, disfrazado con las ropas de Doña Leonor. El hispanista norteamericano Elías L. Rivers escribía en un artículo de 1971 que «cuando una monja jerónima del siglo XVII, en el Méjico de los virreyes, se atreve a escribir una comedia de enredo amoroso, nos extraña la libertad con que trata ciertos temas» ${ }^{4}$.

Sé que el equivoco que permite las palabras de amor cruzadas entre Don Pedro y Castaño, de claro efecto cómico, se rompe al final de la obra. Sé también que, aunque no muy frecuentemente, alguna otra vez aparecen en el teatro clásico español hombres vestidos de mujer: así en Cornelia, de Juan de Timoneda, o en La negra por el honor, de Agustín Moreto. Incluso en representaciones cortesanas podían darse tales disfraces. Un jesuita describe una de ellas en una carta. Leemos, entre otras cosas, que el rey hizo de ayuda de cámara viejo y que el príncipe representó al Duque de Pastrana, además, «subieron vestidos de alabarderos a lo tudesco el Conde de Oropesa, el Conde de aguilar, el Marqués de la Guarda, D. Francisco de Luzón, y otros; de gentiles-hombres, el Conde Puñoenrostro, el Duque de Híjar, etc; de dueños D. Jaime de Cárdenas, Don Francisco Cisneros, etc; de damas, el Almirante, el Conde de Grajal, el Conde de Villalba, el Marqués de Aytona, etc» ${ }^{5}$. Lo que choca, sin embargo, en Los empeños de una ca$s a$ es que el hombre vestido de mujer no resulte castigado, sino al contrario, y que, mientras se vista (y se viste en escena), se dirija a las espectadoras haciendo gestos afeminados. Así, como Castaño se ha despojado de capa, espada y sombrero y comienza a acicalarse, dice:

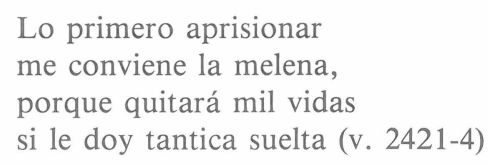

En los versos siguientes (que exigen recitarse con voz atiplada) el gracioso habla aplicándose a sí mismo el género femenino:

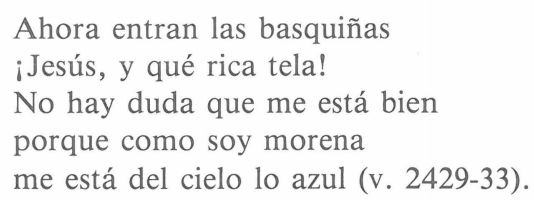

Unos ejemplos más:

Un serenero he topado en aquesta faltriquera.

También me lo he de plantar

4 Elías L. Rivers; «Indecencias de una monjita mejicana»; en Aa, Vv.; Homenaje a W. Fichter; Madrid, Castalia, 1971, pp. 633-637.

5 Carmen Bravo Villasante; La mujer vestida de hombre en el teatro español; Madrid; SGEL, $1976\left(2^{\text {a }}\right)$, pp. $156 / 157$, nota 4. 
¿Cabráme esta pechuguera?

¿Qué les parece señoras

este encaje de ballenas?

Es cierto que estoy hermosa

¡Dios me guarde que estoy bella!

Cualquier cosa me está bien, porque el molde es rara pieza.

Quiero acabar de aliñarme, que aún no estoy dama perfecta.

El manto lo vale todo, échomelo a la cabeza.

¡Válgame Dios! Cuánto encubre esta telilla de seda.

Un trasunto de abanillo

es de mi garbo y belleza, pero si me da tanto aire, ¿qué mucho a mí se parezca? (v. entre 2437 y 2478)

Nos queda la duda de si el actor, en las representaciones, simulaba a lo largo de toda la obra el afeminamiento.

Volvamos al gráfico. En $\mathrm{M}_{1}$ situamos el matrimonio de Don Carlos y Doña Leonor, los dos personajes más sensatos, según todos los críticos. También ahí puede estar el matrimonio de Castaño y Celia, matrimonio «gramaticalizado» puesto que viene impuesto por el género dramático. En $\mathbf{M}_{2}$ podemos ver, pese a la posible exageración, la relación de Castaño con Don Pedro (nunca, en cualquier caso, hubiera podido ser más explícita, dada la prohibición absoluta de que era objeto el llamado 'pecado nefando').

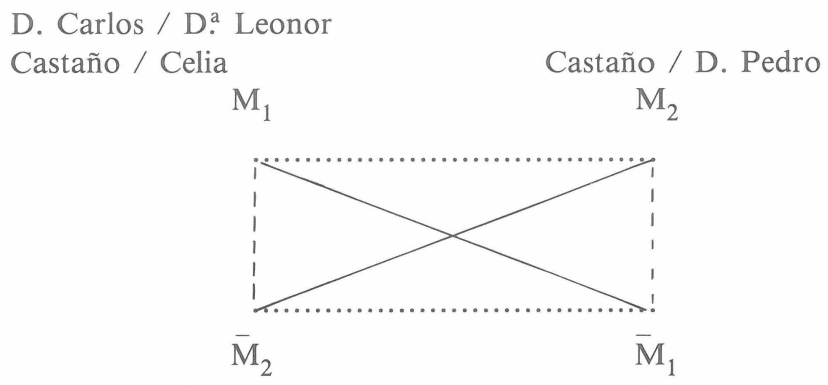

Quedan inconsideradas tres relaciones que se establecen en la obra y que se resuelven de distinto modo. Una es la mantenida por Don Juan y Doña Ana. Otra es la de Don Pedro y Doña Leonor. Por último está la de Don Carlos y Doña Ana. De las tres, sólo una, la de Don Juan y Doña Ana, culmina en matrimonio.

Los deseos de Don Carlos y Doña Leonor son los causantes de todos los desenlaces, pero el planteamiento de la comedia nos permite pensar casi desde el inicio, en la solución final. Veamos por qué. 
Don Pedro ha hecho que Doña Leonor vaya a parar a su propia casa, bajo la custodia de su hermana. Aunque él ha arrebatado la dama y la ha obligado a alojarse en una vivienda contra su voluntad, el rapto (el hecho de llevarse a una mujer del domicilio paterno) lo ha cometido Don Carlos. La deshonra, para el padre de Doña Leonor, reside en el rapto y la manera de curar la honra consiste en que el raptor (el que sea) se case con la raptada. Tal era el uso y la obligación social.

De cara a la publicidad de los hechos Don Pedro ha equivocado su estrategia. Nada hace suponer que sea el raptor, sino un honrado caballero que, debido a la casualidad, decide velar por la honradez de la dama. Como tal, no puede socialmente pretender a la mujer y confesarlo todo sería aparecer como un bellaco. Ha quedado prisionero de su propia ficción. Las relaciones matrimoniales con Doña Leonor quedan, por lo tanto y al contrario de que él pretendía, excluidas. Serán relaciones no matrimoniales $\left(\mathrm{M}_{1}\right)$, puesto que, en justicia, las matrimoniales corresponden al raptor. Raptor de quien, en uso de su libertad individual, la dama está enamorada.

Doña Ana recibe a dos hombres en su casa. Uno, Don Juan, ha venido sin que ella lo sepa. El otro, Don Carlos, entró porque ella quiso aprovechar la circunstancia para enamorarlo. Socialmente no es lo mismo que un hombre entre ocultándose en una casa a que sea una mujer quien lo oculte. En el primer caso, la mujer no comete falta alguna en el segundo sí. La relación de Doña Ana con Don Juan es normal, la de Doña Ana con Don Carlos es 'no buena'.

Hemos completado el cuadro:

\begin{tabular}{|c|c|}
\hline $\begin{array}{l}\text { RELACIONES PERMITIDAS } \\
\text { prescritas: (buenas) } \\
\text { matrimoniales }\end{array}$ & $\begin{array}{r}\text { RELACIONES EXCLUIDAS } \\
\text { prohibidas: (malas) } \\
\text { anormales }\end{array}$ \\
\hline $\begin{array}{l}\text { D. Carlos / Da Leonor } \\
\text { Castaño / Celia } \\
\qquad M_{1}\end{array}$ & $\begin{array}{l}\text { Castaño / D. Pedro } \\
\mathrm{M}_{2}\end{array}$ \\
\hline$\overline{\mathrm{M}}_{2}$ & $\overline{\mathrm{M}}_{1}$ \\
\hline D. Juan / D. Ana & $\begin{array}{l}\text { D. Pedro / Da Leonor } \\
\text { D. Carlos / D. Ana }\end{array}$ \\
\hline $\begin{array}{l}\text { no prohibidas: (no malas) } \\
\text { normales }\end{array}$ & $\begin{array}{r}\text { no prescritas: (no buenas) } \\
\text { no matrimoniales }\end{array}$ \\
\hline
\end{tabular}

El modelo no es plenamente comprensible si no lo superponemos al que corresponde a los deseos de los individuos. En este segundo las relaciones matrimoniales se interpretan como deseadas, componiéndose el cuadrado semiótico del siguiente modo: 


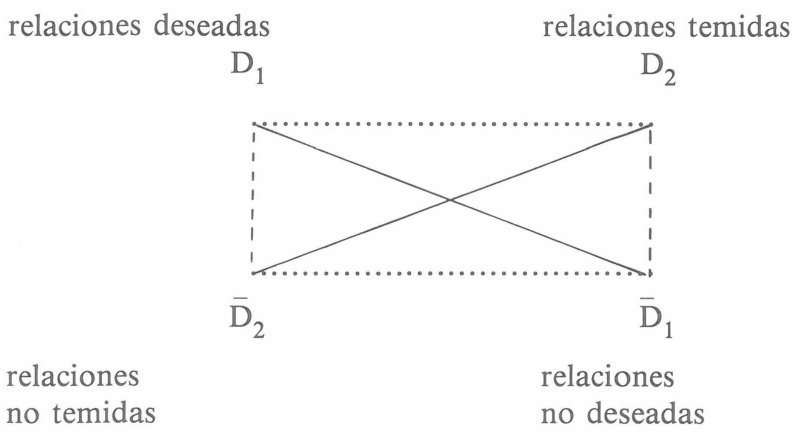

En $M_{1}$ la situación es deseada tanto para Don Carlos y Doña Leonor como para Castaño y Celia. Es producto de la suma $\mathrm{D}_{1}+\mathrm{D}_{1}$, en ambos matrimonios. Es una relación que llamaremos 'equilibrada'. El mismo tipo de relación se da en $\mathrm{M}_{2}$, puesto que es producto de $\mathrm{D}_{2}+\mathrm{D}_{2}$ : no la desean ni Castaño ni Don Pedro.

En $\bar{M}_{2}$, Don Juan desea el matrimonio, pero no Doña Ana, aunque no lo teme, ya que son ambos de la misma clase social. La suma, pues, es producto de $\mathrm{D}_{1}+\mathrm{D}_{2}$, en relación que llamaremos 'compatible' puesto que ambos sumandos se hallan en implicación.

Ahora bien, si don Pedro ama a Doña Leonor $\left(D_{1}\right)$, no puede conseguirla porque ella no lo desea y debe casarse con Don Carlos $\left(\mathrm{D}_{1}\right)$. Es una relación entre contradictorios y, por ello, resulta incompatible. Igual sucede en el caso de la posible entre Don Carlos y Doña Ana.

En este análisis de la estructura del contenido se ha buscado y seguido una isotopía semántica; es decir: la repetición de determinadas unidades a lo largo del discurso. En este caso se trataba de las isotopías que indican las relaciones hombre / mujer tendentes al matrimonio. Para elegir dicha línea isotópica se tuvo en cuenta una diversidad de condicionamientos culturales manifestados como prescripciones, prohibiciones o deseos. Ello ha permitido descubrir en la comedia una serie de enlaces que denominamos equilibrados, compatibles o incompatibles.

El enlace o relación equilibrada se produce cuando los implicados están de acuerdo y se resuelve en la dirección deseada por ellos. La relación compatible se resuelve según los usos sociales y el código del género literario. También los códigos marcan las relaciones incompatibles.

El feminismo de Sor Juana ha servido, probablemente, para que el deseo amoroso y el deseo social coincidan sin grandes conflictos. No puede emparejarse esta obra con otras del Siglo de Oro, apologías del tipo de educación que conviene a la mujer: la que lleva al matrimonio ${ }^{6}$. Tampoco con aquéllas en las que el honor y no el amor organiza el emparejamiento final. La defensa que hace de la dama culta es un buen ejemplo. Aquí el matrimonio se desea o se acepta porque, casada la persona que más se deseaba, no contradice los sentimientos íntimos. Nada se ha forzado. Calderón sirvió de modelo, pero sólo de modelo formal.

6 Véase Aurora Egido: «La universidad del amor y La dama boba»; en Boletín de la biblioteca Menéndez Pelayo LIV, 1978. 
